\title{
What does a rate in a mean ergodic theorem imply?
}

\author{
AlEXANDER GOMILKO AND YURI TOMILOV
}

\begin{abstract}
We develop a general framework for the inverse mean ergodic theorems with rates for operator semigroups, thus completing a construction in the theory initiated in [17] and [18].
\end{abstract}

Mathematics Subject Classification (2010): 47A60 (primary); 47A35, 47D03 (secondary).

\section{Introduction}

In this paper we are concerned with the rates of convergence of Cesáro means

$$
\mathrm{C}_{t}(A):=\frac{1}{t} \int_{0}^{t} T(s) d s, \quad t>0,
$$

for a bounded $C_{0}$-semigroup $(T(t))_{t \geq 0}$ with generator $-A$ on a (complex) Banach space $X$. Recall that in general

$$
\left\{x \in X: \mathrm{C}_{t}(A) x \text { strongly converges }\right\}=\operatorname{ker}(A) \oplus \overline{\operatorname{ran}}(A) .
$$

Moreover, $\mathrm{C}_{t}(A) x$ converges to zero if and only if $x \in \overline{\operatorname{ran}}(A)$.

A mean ergodic theorem provides conditions under which the means in (1.1) converge strongly on the whole of $X$. One of the most well-known mean ergodic theorems says that if $X$ is reflexive then

$$
X=\operatorname{ker}(A) \oplus \overline{\operatorname{ran}}(A)
$$

hence $\mathrm{C}_{t}(A), t>0$, are strongly convergent. Mean ergodic theorems are a classical chapter of ergodic theory, and for its basic results one may consult [27] and [21].

If a mean ergodic theorem holds then it is natural to try to equip it with a certain convergence rate. After a simple normalization, one can assume without loss of generality that $\mathrm{C}_{t}(A), t>0$, converge to zero as $t \rightarrow \infty$. Thus we will

The authors were partially supported by the NCN grant DEC-2011/03/B/ST1/00407.

Received February 19, 2013; accepted in revised form December 20, 2013. 
study the decay rates of $\left\|\mathrm{C}_{t}(A) x\right\|, x \in X$. (See the introduction of [18] for a more detailed discussion.)

The rates in ergodic theorems were studied in many settings and backgrounds. For some of the achievements in this area one may consult the survey papers [ 3 , 25] (and the references therein), and also [1,7-15, 26, 29] and [33]. However no systematic approach to characterizing rates in mean ergodic theorems was proposed until very recent time. The present paper provides one more step towards such a characterization. It is a companion to our previous articles [17] and [18] where the theory of rates in mean ergodic theorems was developed by methods of functional calculus. It was our initial idea that a functional calculus approach might produce certain rates of decay of Cesáro means in a canonical way and thus would allow us to quantify their convergence properties. This idea appeared to be fruitful and opened a door to many tools from outside of ergodic theory. Taking advantage of these new tools we are now able to introduce and study in detail abstract inverse theorems on decay rates, the main subject of this paper.

To set the scene, let us first recall certain direct theorems on rates obtained in [17] and [18]. The direct problem in the study of rates for Cesáro means can be formulated as follows:

Direct Problem: Given $x$ from the range (or the domain) of a function of $A$ find a rate of decay (if any) for $\mathrm{C}_{t}(A) x$ and prove its optimality. (Of course, we should specify what we mean by "function" and "optimality", and this will be clear from further considerations.)

Theorems answering the direct problem will be called direct mean ergodic theorems with rates. Motivated by probabilistic applications, the problem of obtaining various direct theorems with rates has attracted considerable attention during last years. We note the foundational paper [16] and then the subsequent papers [1], [7-15,26].

Recently, we proposed in [17] and [18] an abstract framework which allowed us to encompass many partial results and to solve certain open problems on the rates of decay of Cesáro means. In particular, we proved in [18, Theorem 3.4 and Proposition 4.2] that if $(T(t))_{t \geq 0}$ is a bounded $C_{0}$-semigroup on $X$ and $f$ is a Bernstein function with $\lim _{t \rightarrow 0+} f(t)=0$, then

$$
x \in \operatorname{ran}(f(A)) \Longrightarrow\left\|\mathrm{C}_{t}(A) x\right\|=\mathrm{O}\left(f\left(t^{-1}\right)\right), \quad t \rightarrow \infty .
$$

As corollaries, we obtained rates of decay of Cesáro means on the ranges of polynomial and logarithmic functions, thus extending and sharpening known results. Our results were proved to be optimal in a natural sense.

To understand the limitations of direct mean ergodic theorems with rates it is natural to ask whether the implication (1.3) can be reversed. Examples show that one cannot in general expect the implication opposite to (1.3) to be true (see e.g. Section 4 of the present paper and [16, Example, page 121] concerning the discrete setting). Thus we are interested in the best possible conditions on the decay of the means implying the converses of (1.3), and our abstract inverse problem reads as follows: 
Inverse Problem: Given the rate of decay of $\mathrm{C}_{t}(A) x$ for an element $x \in X$ prove that $x$ is in an appropriate range (or domain) of a function of $A$ and show optimality of the result.

Statements of this form will be called inverse mean ergodic theorems with rates. The first inverse theorems were proved in the discrete setting by Browder [2] and Butzer and Westphal [4]. They showed (indirectly in the first case) that if $X$ is reflexive and $T$ is a power bounded operator on $X$, then $\left\|1 / n \sum_{k=0}^{n-1} T^{k} x\right\|=$ $\mathrm{O}(1 / n)$ implies that $x \in \operatorname{ran}(I-T)$. It was also noted in [4] that one cannot produce better rates than $1 / n$, since $\left\|1 / n \sum_{k=0}^{n-1} T^{k} x\right\|=\mathrm{o}(1 / n)$ implies $x=0$. Thus one has to deal with rates between $1 / n$ and $\mathrm{o}(1)$, and the same is true for the continuous time means $\mathrm{C}_{t}(A) x$ when the rate $1 / n$ is replaced by $1 / t$ - see [18] for a discussion. This complicates the study of rates since many plausible conditions involving rates appear to be too strong in view of the extremal $1 / n$ (or $1 / t$ ) property. See $e$.g. our Appendix.

Various partial situations (mostly of polynomial rates and mostly in the discrete framework) were considered in $[6,12,13,16]$, and [26]. The main goal of the present paper is to provide an abstract set-up for the inverse theorems and to give them a systematic treatment. This set-up appears to be coherent with direct theorems obtained in [18] and it constitutes in a sense a final block of the theory developed in [18]. As in the case of direct theorems treated in [18], known inverse theorems on rates for particular cases (e.g. for polynomial rates) can be included in our framework.

In [18] our direct ergodic theorems involved the ranges of complete Bernstein functions of semigroup generators (as e.g. in (1.3)). In the present study of the inverse theorems, it is convenient to restrict attention to the class of Stieltjes functions and to deal with their domains rather than the ranges of (reciprocal) operator complete Bernstein functions. Thus, (1.3) takes the form

$$
x \in \operatorname{dom}(g(A)) \Longrightarrow\left\|\mathrm{C}_{t}(A) x\right\|=\mathrm{O}\left(1 / g\left(t^{-1}\right)\right), \quad t \rightarrow \infty,
$$

for a Stieltjes function $g=1 / f$. Such a setting enables us to apply an (adapted) abstract characterization of the domains of operator complete Bernstein functions due to Hirsch [23]. (A result similar to Hirsch's one was obtained later by R. Schilling, see e.g. in [31, Theorem 12.19, Remark 12.20, and Corollary 12.21] and also [30].)

The paper is based on ideas worked out in [17, 18], and [20]. However, its finer details are essentially different from the arguments used in those papers and it complements the results obtained in $[17,18]$, and [20]. To give a flavor of inverse theorems proved by our technique we indicate a partial converse of the direct theorem formulated above. It illustrates our approach of adding an 'extra rate' to the decay of the means in order to invert the direct statements.

Assume that $\overline{\operatorname{ran}}(A)=X$. If $g$ is a Stieltjes function of the form

$$
g(z)=\int_{0+}^{\infty} \frac{\mu(d s)}{z+s}, \quad z>0,
$$


where $\mu$ is a (non-negative) Radon measure on $(0, \infty)$ such that

$$
\int_{0+}^{\infty} \frac{\mu(d s)}{1+s}<\infty
$$

$g(0+)=\infty$, and $x \in X$ satisfies

$$
\int_{1}^{\infty} \frac{g(1 / t)\left\|\mathrm{C}_{t}(A) x\right\|}{t} d t<\infty,
$$

then $x \in \operatorname{dom}(g(A))$, or, equivalently, $x \in \operatorname{ran}([1 / g](A))$.

Note that there are close relations between inverse mean ergodic theorems for bounded $C_{0}$-semigroups $(T(t))_{t \geq 0}$ and bounded discrete semigroup $\left(T^{n}\right)_{n \geq 0}$, and our approach, in fact, unifies continuous and discrete frameworks. It allows one to study the continuous and the discrete cases simultaneously and to obtain results parallel in spirit and proofs. However, because of space limitations, the functional calculus approach to inverse mean ergodic theorems in the discrete case will be presented elsewhere.

We also show that our statements are sharp and cannot in general be improved. In fact, it appears that they are are optimal even for a very simple multiplication operator on an $L^{1}$ space. However, even in this simple case, there are nontrivial technical difficulties to overcome. Thus a substantial part of the paper is devoted to proving optimality of our results in various senses.

Our Appendix addresses important problems related to inverse theorems, which however stay a bit aside from the mainstream of the exposition, and thus were shifted to a separate part. We believe that it is of independent interest. There we prove that the means cannot be too small in an "integral" sense.

\subsection{Some notation and definitions}

For a closed linear operator $A$ on a complex Banach space $X$ we denote by $\operatorname{dom}(A)$, $\operatorname{ran}(A), \operatorname{ker}(A)$, and $\rho(A)$ the domain, the range, the kernel, and the resolvent set of $A$, respectively. The norm-closure of the range is written as $\overline{\operatorname{ran}}(A)$. The space of bounded linear operators on $X$ is denoted by $\mathcal{L}(X)$. Finally, we set $\mathbb{R}_{+}:=[0, \infty)$ and $\mathbb{C}_{+}:=\{\lambda \in \mathbb{C}: \operatorname{Re} \lambda>0\}$.

ACKNOWLEDGements. The authors are grateful to M. Haase and M. Lin for useful remarks and fruitful discussions. They would also like to thank M. Lin for sending them the unpublished manuscript [6]. Finally, the authors are grateful to the referee for his/her pertinent comments and suggestions. 


\section{Preliminaries}

\subsection{Functional calculus: Bernstein and Stieltjes functions}

In this subsection we recall basic properties of operator Bernstein and Stieltjes functions and prove several auxiliary statements on functional calculi useful for the sequel. Moreover we arrange the material in the way most suitable for our purposes. The machinery developed will be used intensively in the next sections. A discussion of Bernstein and Stieltjes functions and their relevance for functional calculi can also be found in [18, Section 2].

Let $\mathrm{M}\left(\mathbb{R}_{+}\right)$be the Banach algebra of bounded Radon measures on $\mathbb{R}_{+}$. Define the Laplace transform of $\mu \in \mathrm{M}\left(\mathbb{R}_{+}\right)$as

$$
(\mathcal{L} \mu)(z):=\int_{\mathbb{R}_{+}} e^{-s z} \mu(\mathrm{d} s), \quad z \in \mathbb{C}_{+},
$$

and note that $\mathcal{L} \mu$ extends to a continuous function on $\overline{\mathbb{C}}_{+}$. Note that the space

$$
\mathrm{A}_{+}^{1}\left(\mathbb{C}_{+}\right):=\left\{\mathcal{L} \mu: \mu \in \mathrm{M}\left(\mathbb{R}_{+}\right)\right\}
$$

is a commutative Banach algebra with pointwise multiplication and with respect to the norm

$$
\|\mathcal{L} \mu\|_{\mathrm{A}_{+}^{1}}:=\|\mu\|_{\mathrm{M}\left(\mathbb{R}_{+}\right)}=|\mu|\left(\mathbb{R}_{+}\right),
$$

where $|\mu|\left(\mathbb{R}_{+}\right)$denotes the total variation of $\mu$ on $\mathbb{R}_{+}$, and the Laplace transform

$$
\mathcal{L}: \mathrm{M}\left(\mathbb{R}_{+}\right) \longrightarrow \mathrm{A}_{+}^{1}\left(\mathbb{C}_{+}\right)
$$

is an isometric isomorphism.

Let $-A$ be the generator of a bounded $C_{0}$-semigroup $(T(s))_{s \geq 0}$ on a Banach space $X$. Then the mapping

$$
g=\mathcal{L} \mu=\int_{\mathbb{R}_{+}} e^{-s \cdot} \mu(\mathrm{d} s) \mapsto g(A):=\int_{\mathbb{R}_{+}} T(s) \mu(\mathrm{d} s)
$$

(where the integral converges in the strong topology) is a continuous algebra homomorphism of $\mathrm{A}_{+}^{1}\left(\mathbb{C}_{+}\right)$into $\mathcal{L}(X)$. The homomorphism is called the Hille-Phillips (HP-) functional calculus for $A$. Its basic properties can be found in [21, Chapter $\mathrm{XV}]$.

The $H P$-calculus has an extension to a larger function class. This extension is constructed as follows: if $f: \mathbb{C}_{+} \rightarrow \mathbb{C}$ is holomorphic such that there exists a function $e \in \mathrm{A}_{+}^{1}\left(\mathbb{C}_{+}\right)$with $e f \in \mathrm{A}_{+}^{1}\left(\mathbb{C}_{+}\right)$and the operator $e(A)$ is injective, then we define

$$
f(A):=e(A)^{-1}(e f)(A)
$$

with its natural domain $\operatorname{dom}(f(A)):=\{x \in X:(e f)(A) x \in \operatorname{ran}(e(A))\}$. In this case $f$ is called regularizable, and $e$ is called a regularizer for $f$. Such a 
definition of $f(A)$ does not depend on the particular regularizer $e$ and $f(A)$ is a closed operator on $X$. Moreover, the set of all regularizable functions $f$ is an algebra depending on $A$ (see e.g. [19, page 4-5] and [14, page 246-249]), and the mapping

$$
f \longmapsto f(A)
$$

from this algebra into the set of all closed operators on $X$ is called the extended Hille-Phillips calculus for A. The next product rule of this calculus (see e.g. [19, Chapter 1]) will be crucial for the sequel: if $f$ is regularizable and $g \in \mathrm{A}_{+}^{1}\left(\mathbb{C}_{+}\right)$, then

$$
g(A) f(A) \subseteq f(A) g(A)=(f g)(A),
$$

where we take the natural domain for a product of operators.

This regularization approach can be applied to the study of operator Bernstein functions. First recall that $f \in \mathrm{C}^{\infty}(0, \infty)$ is called a complete monotone function if

$$
f(t) \geq 0 \quad \text { and } \quad(-1)^{n} \frac{d^{n} f(t)}{d t^{n}} \geq 0 \quad \text { for all } n \in \mathbb{N} \text { and } t>0 .
$$

A positive function $f \in \mathrm{C}^{\infty}(0, \infty)$ is called Bernstein function if its derivative is completely monotone. By [31, Theorem 3.2], $f$ is Bernstein if and only if there exist constants $a, b \geq 0$ and a positive Radon measure $\mu$ on $(0, \infty)$ satisfying

$$
\int_{0+}^{\infty} \frac{s}{1+s} \mu(d s)<\infty
$$

and such that

$$
f(z)=a+b z+\int_{0+}^{\infty}\left(1-e^{-s z}\right) \mu(d s), \quad z>0 .
$$

Formula (2.3) is called the Levy-Khintchine representation of $f$. The triple $(a, b, \mu)$ is uniquely determined by the corresponding Bernstein function $f$ and is called the Levi-Khintchine triple.

It was proved in [18, Lemma 2.5] that Bernstein functions belong to the extended HP-functional calculus and every Bernstein function is regularizable by any of the functions $e_{\lambda}(z)=(\lambda+z)^{-1}, \operatorname{Re} \lambda>0$. This led to the following operator Levy-Khintchine representation for a Bernstein function $f$ of $A(c f$. (2.3)) essentially due to Phillips [28].

Theorem 2.1. Let $-A$ generate a bounded $C_{0}$-semigroup $(T(s))_{s \geq 0}$ on $X$, and let $f \sim(a, b, \mu)$ be a Bernstein function. Then $f(A)$ is defined in the extended HPcalculus. Moreover, $\operatorname{dom}(A) \subseteq \operatorname{dom}(f(A))$ and

$$
f(A) x=a x+b A x+\int_{0+}^{\infty}(I-T(s)) x \mu(d s)
$$

for each $x \in \operatorname{dom}(A)$, and $\operatorname{dom}(A)$ is a core for $f(A)$. If $a>0$, then $\operatorname{ran}(f(A))=$ $X$ and $f(A)$ is invertible.

For the detailed theory of operator Bernstein functions we refer to [31]. 
The class of Bernstein functions is quite large and to ensure good algebraic and function-theoretic properties of Bersntein functions it is convenient and also sufficient for many purposes to consider its subclass consisting of complete Bernstein functions. A Bernstein function is called complete if its representing measure in the Levy-Khintchine formula (2.3) has a completely monotone density with respect to Lebesgue measure, see [31, Definition 6.1].

To discuss other representations of complete Bernstein functions, more suitable for the goals of this paper, we will also need yet another related class of functions. A function $g:(0, \infty) \rightarrow \mathbb{R}_{+}$is called Stieltjes if it can be written as

$$
g(z)=a+\frac{b}{z}+\int_{0+}^{\infty} \frac{\mu(d s)}{z+s}, \quad z>0,
$$

where $a, b \geq 0$ and $\mu$ is a positive Radon measure on $(0, \infty)$ satisfying

$$
\int_{0+}^{\infty} \frac{\mu(d s)}{1+s}<\infty
$$

Since the representation (2.5) is unique, the measure $\mu$ is called a Stieltjes measure for $g$ and (2.5) is called the Stieltjes representation for $g$, see e.g. [31, Chapter 2]. We will then write $g \sim(a, b, \mu)$. Note that

$$
a=g(\infty), \quad b=\lim _{z \rightarrow 0+} z g(z) .
$$

The following result (see [31, Theorem 6.2 and Corollary 7.4]) shows, in particular, a reciprocal duality between complete Bernstein and Stieltjes functions, and it will be crucial for the sequel.

Theorem 2.2. A non-zero function $g$ is a Stieltjes function if and only $z g(z), z>0$, is a complete Bernstein function if and only if $1 / \mathrm{g}$ is a complete Bernstein function.

Remark 2.3. Thus every complete Bernstein function $f$ admits a unique representation

$$
f(z)=a+b z+\int_{0+}^{\infty} \frac{z}{z+s} \mu(d s), \quad z>0,
$$

where $a, b \geq 0$ and $\mu$ is a positive Radon measure on $(0, \infty)$ satisfying $(2.6)$, and we can speak of the Stieltjes representation $(a, b, \mu)$ of $f$, and write $f \sim(a, b, \mu)$.

However, there are also other representations for complete Bernstein functions in the literature. For example, we note the representation

$$
f(z)=a+b z+\int_{0+}^{\infty} \frac{z v(d s)}{1+z s}, \quad \int_{0+}^{\infty} \frac{v(d s)}{1+s}<\infty,
$$

used in particular in [22] and [23]. Representations (2.8) and (2.9) are equivalent in the sense that one of them is transformed by the change of variable $s=1 / t$ into another so that the measures $\mu$ and $v$ satisfy the same integrability condition (2.6). 
We will be interested in Stieltjes functions $g$ with the Stieltjes representation of the form $(0,0, \mu)$, and satisfying $g(0+)=\infty$. Note the latter condition which will be crucial for the sequel. Before going further, we give several elementary examples of such functions important for the sequel.

\section{Example 2.4.}

a) The functions $g_{\gamma}(z):=z^{-\gamma}, \gamma \in(0,1)$, are Stieltjes and

$$
\lim _{s \rightarrow 0+} g(s)=\infty, \quad g_{\gamma}(z)=\frac{\sin \pi \gamma}{\pi} \int_{0}^{\infty} \frac{d s}{(z+s) s^{\gamma}}, \quad z>0
$$

Accordingly, $f_{\gamma}(z)=z g_{\gamma}(z)=z^{1-\gamma}, \gamma \in(0,1)$, are complete Bernstein functions.

b) By [18, Example 2.9] the function

$$
g(z):=\frac{\log z}{z-1}=\int_{0}^{\infty} \frac{d s}{(z+s)(1+s)}, \quad z>0,
$$

is Stieltjes with $g(0+)=\infty$, and so is the function $g(z):=\frac{z-1}{z \log z}$ by Theorem 2.2.

Let us show now that complete Bernstein and Stieltjes functions of the generator $-A$ can be expressed in resolvent terms in accordance with the formulas (2.5) and (2.8). To this aim, we will need the notion of a sectorial operator. Recall that a linear operator $V$ on $X$ is called sectorial if $(-\infty, 0) \subset \rho(V)$ and there exists $c>0$ such that

$$
s\left\|(s+V)^{-1}\right\| \leq c, \quad s>0 .
$$

Theorem 2.5. Let $-A$ be the generator of a bounded $C_{0}$-semigroup on $X$.

(i) If $f \sim(0,0, v)$ is a complete Bernstein function, then

$$
f(A) x=\int_{0+}^{\infty} A(s+A)^{-1} x v(d s)
$$

for every $x \in \operatorname{dom}(A)$. Moreover, $\operatorname{dom}(A)$ is a core for $f(A)$.

(ii) If $g \sim(0,0, \mu)$ is a Stieltjes function and $A$ has dense range, then $g$ belongs to the extended HP-calculus and

$$
g(A) x=\int_{0+}^{\infty}(s+A)^{-1} x \mu(d s)
$$

for every $x \in \operatorname{ran}(A)$. Moreover, $\operatorname{ran}(A)$ is a core for $g(A)$. 
Proof. The proof of (2.10) relies on a direct transforming of (2.4) to the form (2.10) by means of the definition of a complete Bernstein function and can be found in [31, page 149]. The fact that $\operatorname{dom}(A)$ is a core for $f(A)$ follows from Theorem 2.1. Thus (i) is a straightforward consequence of Theorem 2.1.

To prove (ii) note that by Theorem 2.2 if $g$ is a Stieltjes function then $g(z)=$ $q(z) / z$ for some complete Bernstein function $q$. Since by [18, Lemma 2.5] $q$ is regularizable by $1 /(z+1)$ and $A$ is injective in view of (1.2), the function $g$ is regularizable by $z /(z+1)$ and belongs to the extended HP-calculus. Hence, if $x \in \operatorname{ran}(A)$, then using the product rule for the extended HP-calculus we obtain

$$
\begin{aligned}
g(A) x & =\left[\frac{z+1}{z} \cdot z g \cdot \frac{1}{z+1}\right](A) x \\
& =(A+I) A^{-1} \int_{0+}^{\infty} A(s+A)^{-1}(A+I)^{-1} x \mu(d s) \\
& =\int_{0+}^{\infty}(s+A)^{-1} x \mu(d s) .
\end{aligned}
$$

It remains to prove that $\operatorname{ran}(A)$ is a core for $g(A)$. Observe that by e.g. [19, Proposition 2.2.1,b] the operator $A^{-1}$ is sectorial with dense domain $\operatorname{ran}(A)$. Hence if $e_{t}(A)=t\left(t+A^{-1}\right)^{-1}, t>0$, then $e_{t}(A) x \rightarrow x$ for every $x \in X$ as $t \rightarrow \infty$ by [19, Proposition 2.2.1, c]. Since $e_{t} \in \mathrm{A}_{+}^{1}\left(\mathbb{C}_{+}\right)$for each $t>0$, the product rule (2.2) implies that if $x \in \operatorname{dom}(g(A))$ and $g(A) x=y$ then $g(A) e_{t}(A) x=e_{t}(A) y$. As $\operatorname{ran}\left(e_{t}(A)\right)=\operatorname{dom}\left(A^{-1}\right)=\operatorname{ran}(A)$, the statement follows.

Let $g$ be a Stieltjes function with the Stieltjes representation $(0,0, \mu)$, i.e.

$$
g(z)=\int_{0+}^{\infty} \frac{\mu(d s)}{z+s}, \quad z>0, \quad \int_{0+}^{\infty} \frac{\mu(d s)}{1+s}<\infty .
$$

If a complete Bernstein function $h$ is given by

$$
h(z):=g(1 / z)=\int_{0+}^{\infty} \frac{z \mu(d s)}{1+z s}
$$

and a linear operator $V$ is sectorial then the operator $h(V)$ can be defined as the closure of a (closable) linear operator $h_{0}(V)$ given by the formula

$$
h_{0}(V) x=\int_{0+}^{\infty} V(1+s V)^{-1} x \mu(d s), \quad x \in \operatorname{dom}(V) .
$$

This definition is due to Hirsch and it was introduced and thoroughly studied in his paper [22]. Thus $h(V)$ is a closed linear operator and $\operatorname{dom}(h(V)) \supset \operatorname{dom}(V)$. A simple change of variables shows that (2.14) takes the form of (2.10) with $A=V$ (and, in general, a different Stieltjes measure.) Since the mapping $g(z) \rightarrow g(1 / z)$ is a bijection from the set of Stieltjes functions onto the set of complete Bersntein 
functions, Hirsch's definition (2.14) extends (2.10) to complete Bernstein functions $f \sim(0,0, v)$ of sectorial operators $A$.

If $A^{-1}$ is a sectorial operator with dense domain $\operatorname{ran}(A)$, then setting $V=A^{-1}$ in (2.14) we obtain

$$
h\left(A^{-1}\right) x=\int_{0+}^{\infty}(A+s)^{-1} x \mu(d s)=g(A) x, \quad x \in \operatorname{ran}(A) .
$$

Hence the operators $h\left(A^{-1}\right)$ and $g(A)$ coincide on their core $\operatorname{dom}\left(A^{-1}\right)=\operatorname{ran}(A)$ and therefore coincide. In other words, $g(A)$ defined in the extended HP-calculus coincides with $h\left(A^{-1}\right)$ defined by means of (2.14).

Hirsch proved in [22] a number of properties of complete Bernstein functions of sectorial operators. We will need two of them which we state as Lemma 2.6. For their proofs see [22, Theorem 1, page 257] and [22, Theorem 3, page 261].

Lemma 2.6. Let $f$ and $q$ be complete Bernstein functions and let A be a sectorial operator with dense range. Then

(i) $f(A)$ is a sectorial operator with dense range;

(ii) $(f \circ q)(A)=f(q(A))$.

The property (2.15) will allow us to link several results from [22] and [23] to our setting of Stieltjes functions of semigroup generators.

Lemma 2.7. Let $-A$ be the generator of a bounded $C_{0}$-semigroup on $X$, with dense range. If $f$ and $g$ as in Theorem 2.5, then their composition $f \circ g$ belongs to the extended HP-calculus, and $f(g(A))=(f \circ g)(A)$, where $f(g(A))$ is defined by means of (2.11) and (2.10). As a consequence,

$$
\operatorname{dom}((f \circ g)(A)) \supset \operatorname{dom}(g(A)) \text {. }
$$

Proof. Note first that $f \circ g$ is a Stieltjes function by [31, Theorem 7.5]. From [24, Lemma 3.9.34 B] and the monotonicity of $g$ it follows that there exists $C=C_{f, g}>$ 0 such that $f(g(t)) \leq C g(t)$ whenever $0<t \leq 1$. Hence, in view of (2.7), we obtain $\lim _{t \rightarrow 0+} t(f \circ g)(t) \leq \lim _{t \rightarrow 0+} t C g(t)=0$, and, since $\lim _{t \rightarrow \infty} g(t)=0$, we also have $\lim _{t \rightarrow \infty} f(g(t))=0$. Thus $f \circ g$ has the Stieltjes representation of the form $(0,0, v)$. By assumption, $A^{-1}$ is sectorial with dense domain. So if $g \sim(0,0, \mu)$ is a Stieltjes function and a complete Bernstein function $h$ is given by (2.13), then $g(A)=h\left(A^{-1}\right)$, where $h\left(A^{-1}\right)$ is defined by means of (2.14). By Lemma 2.6 (i) the operator $h\left(A^{-1}\right)$ is sectorial with dense range, so $g(A)$ is the same.

Then using Lemma 2.6 (ii) and (2.15) we conclude that

$$
f(g(A))=f\left(h\left(A^{-1}\right)\right)=(f \circ h)\left(A^{-1}\right)=[(f \circ h)(1 / z)](A)=(f \circ g)(A) .
$$

Since $\operatorname{dom} f(g(A)) \supset \operatorname{dom}(g(A))$, this implies that $\operatorname{dom}((f \circ g)(A))=\operatorname{dom} f(g(A)) \supset$ $\operatorname{dom}(g(A))$. 
The next statement describing domains of operator Stieltjes functions in resolvent terms is basic for the paper. It is in fact a reformulation of Hirsch's result [23, Theorem 2] by means of (2.15).

Theorem 2.8 (Hirsch Criterion). If $-A$ is the generator of a bounded $C_{0}$-semigroup on $X$ such that $\overline{\operatorname{ran}}(A)=X$ and $g$ is a Stieltjes function given by (2.12), then

$$
\begin{aligned}
x \in \operatorname{dom}(g(A)) & \Longleftrightarrow \exists \text { weak }-\lim _{\delta \rightarrow 0+} \int_{\delta}^{1}(A+s)^{-1} x \mu(d s) \\
& \Longleftrightarrow \exists \lim _{\delta \rightarrow 0+} \int_{\delta}^{1}(A+s)^{-1} x \mu(d s) .
\end{aligned}
$$

Note that if $g \sim(0,0, \mu)$ is a Stieltjes function then

$$
g(t)=\int_{0}^{\infty} e^{-t s} m(s) d s, \quad m(s)=\int_{0}^{\infty} e^{-s \tau} \mu(d \tau), \quad t, s>0,
$$

where $m$ is integrable in the neighborhood of zero and $\lim _{t \rightarrow \infty} m(t)=0$. (In fact, by the Lebesgue bounded convergence theorem, if $g$ is Stieltjes and (2.17) holds, then $g \sim(0,0, \mu)$.) The following result given in [23, Corollaire, pages 214-215] complements Theorem 2.8. It will also be useful in the sequel.

Theorem 2.9. If $-A$ is the generator of a bounded $C_{0}$-semigroup on $X$ such that $\overline{\operatorname{ran}}(A)=X$ and $g$ is a Stieltjes function given by (2.17), then the following statements are equivalent:

(i) $x \in \operatorname{dom}(g(A))$;

(ii) weak- $\lim _{t \rightarrow \infty} \int_{0}^{t} T(s) x m(s) d s$ exists;

(iii) $\lim _{t \rightarrow \infty} \int_{0}^{t} T(s) x m(s) d s$ exists.

It is instructive to consider several direct consequences of Theorem 2.9. The first one provides a continuous analogue of [16, Theorem 2.11].

Remark 2.10. Let $\alpha \in(0,1)$. If $-A$ is the generator of a bounded $C_{0}$-semigroup $(T(t))_{t \geq 0}$ on $X$ and $\overline{\operatorname{ran}}(A)=X$, then, since

$$
z^{-\alpha}=\int_{0}^{\infty} e^{-z s} m(s) d s, \quad m(s)=\frac{s^{\alpha-1}}{\Gamma(\alpha)}, \quad s>0,
$$

we obtain by Theorem 2.9 that $x \in \operatorname{dom}\left(A^{-\alpha}\right)$ if and only if

$$
\lim _{t \rightarrow \infty} \int_{0}^{t} \frac{T(s) x}{s^{1-\alpha}} d s \quad \text { exists, }
$$

in either the weak or the strong topology of $X$. 
The second consequence of Theorem 2.9 can be considered as a continuous counterpart of [5, Theorem 3.9] and [20, Theorem 6.2] where it was proved that

$$
\operatorname{dom}(\log (I-T))=\left\{x: \sum_{n=1}^{\infty} \frac{T^{n} x}{n} \quad \text { converges weakly (strongly) }\right\}
$$

for a power-bounded operator $T$ on $X$.

\section{Remark 2.11.}

a) Note that $g(z):=\log \left(1+z^{-1}\right), z>0$, is Stieltjes and

$$
g(z)=\int_{0}^{1} \frac{d s}{z+s}=\int_{0}^{\infty} e^{-z s} m(s) d s, \quad m(s):=\int_{0}^{1} e^{-s t} d t .
$$

Clearly,

$$
m(s)=\frac{1}{s}+\mathrm{O}\left(e^{-s}\right), \quad s \rightarrow \infty .
$$

By Theorem 2.9, if $-A$ is the generator of a bounded $C_{0}$-semigroup $(T(t))_{t \geq 0}$ on $X$ and $\overline{\operatorname{ran}}(A)=X$, then

$$
x \in \operatorname{dom}\left(\log \left(I+A^{-1}\right)\right) \Longleftrightarrow \lim _{t \rightarrow \infty} \int_{1}^{t} \frac{T(s) x}{s} d s \quad \text { exists }
$$

in either weak or strong topology of $X$.

b) Similarly, $g(z):=\log (z) /(z-1)$ is Stieltjes and

$$
g(z)=\int_{0}^{\infty} e^{-z s} m(s) d s, \quad m(s):=\int_{0}^{\infty} \frac{e^{-t}}{t+s} d t,
$$

see [18, Example 2.9]. By integration by parts,

$$
m(s)=\frac{1}{s}+\mathrm{O}\left(1 / s^{2}\right), \quad s \rightarrow \infty .
$$

Thus, if $-A$ generates a bounded $C_{0}$-semigroup on $X$ and $\overline{\operatorname{ran}}(A)=X$, then

$$
x \in \operatorname{dom}\left(\frac{\log z}{z-1}(A)\right) \Longleftrightarrow \lim _{t \rightarrow \infty} \int_{1}^{t} \frac{T(s) x}{s} d s \text { exists. }
$$

Note that under the above assumptions on $A, \log (A)$ can be defined by means of the extended Hille-Phillips calculus, see [18, Section 5.3]. In view of (2.18) it would be natural to try to replace $\operatorname{dom}\left(\log \left(I+A^{-1}\right)\right)$ in $(2.19)$ by $\operatorname{dom}(\log (A))$. This is however not possible, in general, as the following simple example shows. 
Example 2.12. Let $X=L^{2}(0, \infty)$ and define a selfadjoint positive operator $A$ on $X$ as

$$
(A x)(s):=s x(s), \quad x \in X, \quad s>0 .
$$

It is straightforward that $-A$ generates a contraction $C_{0}$-semigroup $(T(t))_{t \geq 0}$ on $X$ given by

$$
(T(t) x)(s)=e^{-t s} x(s), \quad x \in X, \quad t \geq 0,
$$

and that $A$ has dense range. Since

$$
y \in \operatorname{dom}(\log (A)) \Longleftrightarrow \int_{0}^{\infty}\left(1+\log ^{2}(s)\right)|y(s)|^{2} d s<\infty,
$$

and

$$
y \in \operatorname{dom}\left(\log \left(1+A^{-1}\right)\right) \Longleftrightarrow \int_{0}^{\infty}\left(\log ^{2}\left(1+s^{-1}\right)+1\right)|y(s)|^{2} d s<\infty,
$$

we have

$$
\operatorname{dom}(\log (A)) \subset \operatorname{dom}\left(\log \left(1+A^{-1}\right)\right),
$$

and the inclusion is strict, in general. Consider $e . g$.

$$
x_{0}(t):=1_{[1, \infty)}(\sqrt{s} \log (s+1))^{-1} \in \operatorname{dom}\left(\log \left(I+A^{-1}\right)\right) .
$$

Thus, in view of Remark 2.11, the implication

$$
\lim _{t \rightarrow \infty} \int_{1}^{t} \frac{T(s) x}{s} d s \quad \text { exists } \Rightarrow y \in \operatorname{dom}(\log (A))
$$

is not, in general, true.

\section{Rates in mean ergodic theorem}

For the whole of this section, we make the following assumptions:

$$
\begin{aligned}
& -A \text { is the generator of a } C_{0}-\text { semigroup }(T(t))_{t \geq 0}, \\
& C:=\sup _{t \geq 0}\|T(t)\|<\infty, \text { and } \overline{\operatorname{ran}}(A)=X,
\end{aligned}
$$

(recall that in this case, by $(1.2), \operatorname{ker}(A)=\{0\})$. Moreover, we will be considering functions $g$ such that

$$
g \text { is a Stieltjes function, } g \sim(0,0, \mu), \quad g(0+):=\infty .
$$

Let us comment on the above conditions on $g$. For technical reasons, it will be more convenient for us to consider Stieltjes functions as above than those of the 
general form (2.5), (2.6). To see that we do not loose generality this way, note that we may assume $a=0$ in (2.5)(that is $\lim _{s \rightarrow \infty} g(s)=0$ ) since $\operatorname{dom}(g(A)+a)=$ $\operatorname{dom}(g(A))$. If $b \neq 0$ in (2.5) then passing to the reciprocal complete Bernstein function $1 / g$ and using [31, Corollary 12.7] we infer that $\operatorname{dom}(g(A))=\operatorname{ran}(A)$, so that the Cesáro means $\mathrm{C}_{t}(A) x$ for $x \in \operatorname{dom}(g(A))$ decay at the extremal rate:

$$
\left\|\mathrm{C}_{t}(A) x\right\|=\mathrm{O}\left(t^{-1}\right), \quad t \rightarrow \infty, \quad x \in \operatorname{dom}(g(A)) .
$$

The inverse theorems given below become void in this case, see Remark 5.3 in the Appendix. Finally, if $g(0+)<\infty$, then the direct mean ergodic theorems with rates (see (1.4) and also Theorem 3.2 below) do not yield any rate of decay of $\mathrm{C}_{t}(A)$ restricted to $\operatorname{dom}(g(A))$ since $1 / g(1 / t) \nrightarrow \rightarrow 0, t \rightarrow \infty$ in this case.

We start with an elementary inequality.

Lemma 3.1. Let $f \sim(0,0, \mu)$ be a complete Bernstein function. Then

$$
\frac{1}{t} \int_{0+}^{\infty} \frac{1-e^{-s t}}{s} \mu(d s) \leq 2 f\left(t^{-1}\right), \quad t>0 .
$$

Proof. Since

$$
\frac{1-e^{-x}}{x} \leq \frac{2}{1+x}, \quad x>0
$$

we have

$$
\frac{1-e^{-s t}}{t s} \leq \frac{2}{1+t s}=\frac{2 t^{-1}}{t^{-1}+s}, \quad s, t>0
$$

Hence

$$
\frac{1}{t} \int_{0+}^{\infty} \frac{1-e^{-s t}}{s} \mu(d s) \leq 2 \int_{0+}^{\infty} \frac{t^{-1} \mu(d s)}{t^{-1}+s}=2 f\left(t^{-1}\right), \quad t>0 .
$$

We first we derive a convergence rate for $\mathrm{C}_{t}(A) x$ for $x \in \operatorname{dom}(g(A))$, or equivalently for $x \in \operatorname{ran}([1 / g](A))$ with $1 / g$ being a complete Bernstein function. The following theorem is a partial case of [18, Theorem 3.4 and Proposition 4.2] where the convergence rate was obtained in terms of the limit behavior of $1 / g$ at zero for the whole class of Bernstein functions. However, in the particular situation of complete Bernstein functions, we give an argument which is simpler and more transparent than that from [18]. Moreover, it illustrates nicely our functional calculus approach and makes the presentation self-contained.

Theorem 3.2. If $g$ satisfy (3.1) and $x \in \operatorname{dom}(g(A))$, then

$$
\left\|\mathrm{C}_{t}(A) x\right\| \leq 4 C \frac{\|g(A) x\|}{g\left(t^{-1}\right)}, \quad t>0 .
$$


Proof. Remark first that if $g \not \equiv 0$ a Stieltjes function, then $f=1 / g$ is a complete Bernstein function and by [19, Theorem 1.2.2,d)] one has

$$
(f(A))^{-1}=(1 / f)(A)=g(A),
$$

hence $\operatorname{dom}(g(A))=\operatorname{ran}(f(A))$.

Let $y \in \operatorname{dom}(A) \subset \operatorname{dom}(f(A))$ and $t>0$. Then from (2.10) it follows that

$$
\begin{aligned}
t \mathrm{C}_{t}(A) f(A) y & =\int_{0}^{\infty} \int_{0}^{t} T(\tau) A(A+s)^{-1} y d \tau \mu(d s) \\
& =\int_{0}^{\infty}[1-T(t)](A+s)^{-1} y \mu(d s) .
\end{aligned}
$$

Since

$$
[I-T(t)](A+s)^{-1} y=\int_{t}^{\infty}\left(e^{-s(\tau-t)}-e^{-s \tau}\right) T(\tau) y d \tau+\int_{0}^{t} e^{-s \tau} T(\tau) y d \tau,
$$

we infer that

$$
\begin{aligned}
\left\|[1-T(t))(A+s)^{-1} y\right\| & \leq C\|y\|\left\{\int_{t}^{\infty}\left(e^{-s(\tau-t)}-e^{-s \tau}\right) d \tau+\int_{0}^{t} e^{-s \tau} d \tau\right\} \\
& =2 C\|y\| \cdot \frac{1-e^{-s t}}{s} .
\end{aligned}
$$

Thus using Lemma 3.1 we have

$$
\begin{aligned}
\left\|\mathrm{C}_{t}(A) f(A) y\right\| & \leq 2 C\|y\| \int_{0}^{\infty} \frac{1-e^{-s t}}{t s} \mu(d s) \\
& \leq 4 C f\left(t^{-1}\right)\|y\|, \quad y \in \operatorname{dom}(A) .
\end{aligned}
$$

Since $\operatorname{dom}(A)$ is a core for $f(A)$, by passing to closures in the last inequality, we finally obtain

$$
\left\|\mathrm{C}_{t}(A) f(A) y\right\| \leq 4 C f\left(t^{-1}\right)\|y\|, \quad y \in \operatorname{dom}(f(A)) .
$$

Then (3.5) and (3.6) imply (3.4).

Remark 3.3. Note that Theorem 3.2 can be formulated in terms of complete Bernstein functions as in (3.6). It is this form of (3.4) that we have obtained in [18, Theorem 3.4 and Proposition 4.2].

The following result, Theorem 3.4, is our main inverse theorem for rates. At first glance, its assumptions differ from the conclusions of the (direct) Theorem 3.2. We show however that the result yields several statements which are are "almost" converse of Theorem 3.2. The word "almost" is crucial: we prove that the result is optimal and thus there is an unavoidable, in general, gap between our direct and inverse mean ergodic theorems with rates. 
Theorem 3.4. Let $g$ satisfy (3.1). If $x \in X$ is such that

$$
\int_{1}^{\infty} \frac{\left|g^{\prime}(1 / t)\right|\left\|\mathrm{C}_{t}(A) x\right\|}{t^{2}} d t<\infty
$$

then $x \in \operatorname{dom}(g(A))$.

Proof. Note that for any $s>0$ and $x \in X$

$$
\begin{aligned}
(A+s)^{-1} x & =\int_{0}^{\infty} e^{-s t} T(t) x d t=\int_{0}^{\infty} e^{-s t}\left(\int_{0}^{t} T(\tau) x d \tau\right)^{\prime} d t \\
& =\int_{0}^{\infty} s t e^{-s t} C_{t}(A) x d t .
\end{aligned}
$$

Therefore

$$
\left\|(A+s)^{-1} x\right\| \leq C\|x\|+\int_{1}^{\infty} s t e^{-s t}\left\|C_{t}(A) x\right\| d t
$$

and

$$
\begin{aligned}
\int_{0+}^{1}\left\|(A+s)^{-1} x\right\| \mu(d s) & \leq C\|x\| \int_{0+}^{1} \mu(d s) \\
& +\int_{1}^{\infty}\left(\int_{0+}^{1} s t e^{-s t} \mu(d s)\right)\left\|C_{t}(A) x\right\| d t .
\end{aligned}
$$

To estimate the inner integral observe that for every $\tau \geq 0$

$$
\tau e^{-\tau} \leq \frac{4}{(1+\tau)^{2}}
$$

since

$$
4 e^{\tau}-\tau(1+\tau)^{2}>4\left(\sum_{i=0}^{4} \frac{t^{i}}{i !}\right)-\tau-2 \tau^{2}-\tau^{3}>4+\frac{\tau^{3}}{6}(\tau-2)>0 .
$$

Now using (3.8) with $\tau=t s$ we have

$$
\int_{0+}^{\infty} t s e^{-t s} \mu(d s) \leq 4 \int_{0+}^{\infty} \frac{\mu(d s)}{(1+t s)^{2}}=\frac{4}{t^{2}} \int_{0+}^{\infty} \frac{\mu(d s)}{(1 / t+s)^{2}}=4 \frac{\left|g^{\prime}(1 / t)\right|}{t^{2}} .
$$

Thus

$$
\begin{aligned}
\int_{0+}^{1}\left\|(A+s)^{-1} x\right\| \mu(d s) & \leq C\|x\| \int_{0+}^{1} \mu(d s) \\
& +4 \int_{1}^{\infty} \frac{\left|g^{\prime}(1 / t)\right|}{t^{2}}\left\|C_{t}(A) x\right\| d t<\infty
\end{aligned}
$$

and $x \in \operatorname{dom}(g(A))$ by Hirsch's Theorem 2.8. 
The next direct corollary of Theorem 3.4 is formulated in terms of a norm estimate for $\mathrm{C}_{t}(A)$, thus removing assumptions on the derivative of $g$.

Corollary 3.5. Let $g$ satisfy (3.1). If $x \in X$ and a measurable function $\epsilon:(g(1), \infty) \mapsto(0, \infty)$ are such that

$$
\left\|\mathrm{C}_{t}(A) x\right\|=\mathrm{O}\left(\frac{1}{g(1 / t) \epsilon(g(1 / t))}\right), \quad t \rightarrow \infty, \quad \int_{g(1)}^{\infty} \frac{d \tau}{\tau \epsilon(\tau)}<\infty,
$$

then $x \in \operatorname{dom}(g(A))$.

Proof. If (3.10) holds, then there exists $c>0$ such that

$$
\int_{1}^{\infty} \frac{\left|g^{\prime}(1 / t)\right|\left\|\mathrm{C}_{t}(A) x\right\|}{t^{2}} d t \leq c \int_{1}^{\infty} \frac{d g(1 / t)}{g(1 / t) \epsilon(g(1 / t))}=c \int_{g(1)}^{\infty} \frac{d \tau}{\tau \epsilon(\tau)}<\infty,
$$

and Theorem 3.4 implies $x \in \operatorname{dom}(g(A))$.

Now we derive a corollary of Theorem 3.4 which is almost a converse to Theorem 3.2. It is however strictly weaker than Theorem 3.4 and at the same time it cannot be essentially improved, as we will show in Section 4.

Corollary 3.6. Let $g$ satisfy (3.1). If $x \in X$ is such that

$$
\int_{1}^{\infty} \frac{g(1 / t)\left\|\mathrm{C}_{t}(A) x\right\|}{t} d t<\infty
$$

then $x \in \operatorname{dom}(g(A))$.

Proof. It suffices to observe that

$$
\left|g^{\prime}(\tau)\right|=\int_{0+}^{\infty} \frac{\mu(d s)}{(\tau+s)^{2}} \leq \frac{1}{\tau} \int_{0+}^{\infty} \frac{\mu(d s)}{\tau+s}=\frac{g(\tau)}{\tau}, \quad \tau>0 .
$$

(In fact, a more general estimate is given in [24, Lemma 3.9.34].) The claim follows now from Theorem 3.4.

Remark 3.7. Note that Corollary 3.6 can be formulated in terms of the norm estimates for $\mathrm{C}_{t}(A) x$ rather than an integral condition on $\left\|\mathrm{C}_{t}(A) x\right\|$. Indeed, (3.11) is equivalent to the condition

$$
\left\|\mathrm{C}_{t}(A) x\right\|=\mathrm{O}\left(\frac{1}{\epsilon(t) g(1 / t)}\right), \quad t \rightarrow \infty
$$

where $\epsilon:(1, \infty) \rightarrow(0, \infty)$ is a measurable function satisfying

$$
\int_{1}^{\infty} \frac{d \tau}{\tau \epsilon(\tau)}<\infty
$$


Observe that if $g(z)=z^{-\alpha}, \alpha \in(0,1)$, then (3.10) and (3.13) are, in a sense, equivalent. Indeed, if (3.10) holds then setting $\tilde{\epsilon}(\tau)=\epsilon\left(\tau^{\alpha}\right)$ we have

$$
\int_{1}^{\infty} \frac{d \tau}{\tau \tilde{\epsilon}(\tau)}=\frac{1}{\alpha} \int_{1}^{\infty} \frac{d \tau}{\tau \epsilon(\tau)}
$$

and (3.13) is satisfied with $\epsilon$ replaced by $\tilde{\epsilon}$. Conversely, if (3.13) holds then setting $\tilde{\epsilon}(\tau)=\epsilon\left(\tau^{1 / \alpha}\right)$ we infer that (3.13) is true with $\epsilon$ replaced by $\tilde{\epsilon}$.

Using Remark 3.7 we state now the following straightforward consequence of Corollary 3.5 .

Corollary 3.8. Let $g$ satisfy (3.1). If $x \in X$ and there exists $\alpha \in(0,1)$ such that

$$
\left\|C_{t}(A) x\right\|=\mathrm{O}\left(\frac{1}{g(1 / t) \log ^{1+\alpha}(2+g(1 / t))}\right), \quad t \rightarrow \infty
$$

then $x \in \operatorname{dom}(g(A))$.

Specifying (3.14) for a power function we obtain the domain/range condition for fractional powers of $A$.

Corollary 3.9. If $x \in X$ and there exist $\alpha, \beta \in(0,1)$ such that

$$
\left\|C_{t}(A) x\right\|=\mathrm{O}\left(\frac{1}{t^{\beta} \log ^{1+\alpha} t}\right), \quad t \rightarrow \infty
$$

then $x \in \operatorname{dom}\left(A^{-\beta}\right)=\operatorname{ran}\left(A^{\beta}\right)$.

The next result proposes a different ideology for proving the inverse mean ergodic theorems with rates. To be able to place an element $x$ into $\operatorname{dom}(g(A))$ in the results above we had to add an "extra rate" to the rate $r(t)=(g(1 / t))^{-1}$ of the decay of $\mathrm{C}_{t}(A) x$ obtained in Theorem 3.2. Now, instead of adding an "extra rate", we add an "extra domain" to $\operatorname{dom}(g(A))$. In this way, we will show that $x$ belongs to a slightly larger space than $\operatorname{dom}(g(A))$ under the assumption $\left\|\mathrm{C}_{t}(A) x\right\|=$ $\mathrm{O}\left((g(1 / t))^{-1}\right)$. Note that by Theorem $3.2\left\|\mathrm{C}_{t}(A) x\right\|=\mathrm{O}\left((g(1 / t))^{-1}\right)$ would follow from merely $x \in \operatorname{dom}(g(A))$.

Recall first (from Section 2 ) that if $f \sim(0,0, v)$ is a complete Bernstein function and $g \sim(0,0, \mu)$ is a Stieltjes function, then by $(2.16)$, we have

$$
\operatorname{dom}((f \circ g)(A)) \supset \operatorname{dom}(g(A))
$$

and the inclusion is in general strict. While the assumption $\left\|\mathrm{C}_{t}(A) x\right\|=\mathrm{O}\left((g(1 / t))^{-1}\right)$ does not, in general, imply $x \in \operatorname{dom}(g(A))$ we prove that it does suffices to guarantee $x \in \operatorname{dom}((q \circ g)(A))$ for a large subclass of Bernstein functions $q$. 
Theorem 3.10. Let g satisfy (3.1) and let $q$ be a complete Bernstein function such that

$$
\int_{1}^{\infty} \frac{q(\tau)}{\tau^{2}} d \tau<\infty, \quad \lim _{s \rightarrow 0+} q(s)=0 .
$$

If $x \in X$ satisfies

$$
\left\|\mathrm{C}_{t}(A) x\right\|=\mathrm{O}\left(\frac{1}{g(1 / t)}\right), \quad t \rightarrow \infty
$$

then $x \in \operatorname{dom}((q \circ g)(A))$. In particular, if (3.16) holds, then for any $\alpha \in(0,1)$ one has $x \in \operatorname{dom}\left(\left[g^{\alpha}\right](A)\right)$.

Proof. Since (3.15) implies that $q \sim(0,0, \mu)$, we infer as in the proof of Lemma 2.7 that $q \circ g$ is Stieltjes, and has the Stieltjes representation of the form $(0,0, v)$.

Next we apply Theorem 2.8 to $x$ and $(q \circ g)(A)$. Using the hypothesis on the decay of $\left\|\mathrm{C}_{t}(A) x\right\|$ and the estimate (3.9) from the proof of Theorem 3.4, we obtain

$$
\begin{aligned}
\int_{0+}^{1}\left\|(A+s)^{-1} x\right\| v(d s) \leq & C\|x\| \int_{0+}^{1} v(d s) \\
& +4 c \int_{1}^{\infty} \frac{1}{g(1 / t)} d(q(g(1 / t))
\end{aligned}
$$

for some $c>0$. Furthermore

$$
\begin{aligned}
\int_{1}^{\infty} \frac{1}{g(1 / t)} d(q(g(1 / t)) & =\int_{1}^{\infty} \frac{q^{\prime}(g(1 / t))}{g(1 / t)} d g(1 / t)=\int_{g(1)}^{\infty} \frac{q^{\prime}(\tau)}{\tau} d \tau \\
& =-\frac{q(g(1))}{g(1)}+\int_{g(1)}^{\infty} \frac{q(\tau)}{\tau^{2}} d \tau \leq \int_{g(1)}^{\infty} \frac{q(\tau)}{\tau^{2}} d \tau .
\end{aligned}
$$

Thus finally

$$
\int_{0+}^{1}\left\|(A+s)^{-1} x\right\| v(d s) \leq C\|x\| \int_{0+}^{1} v(d s)+4 c \int_{g(1)}^{\infty} \frac{q(\tau)}{\tau^{2}} d \tau<\infty,
$$

and then $x \in \operatorname{dom}((q \circ g)(A))$. The last statement follows from the fact that $z^{\alpha}, \alpha \in$ $(0,1)$, is a complete Bernstein function satisfying (3.15).

Using (2.17) and our direct mean ergodic theorems with rates, we prove below a characterization of dom $(g(A))$ in terms of $\mathrm{C}_{t}(A)$ which is based on Hirsch's Theorem 2.9. It involves certain means of $\mathrm{C}_{t}(A)$ thus avoiding a need of adding "extra rate" or "extra range". It is however less explicit than Theorems 3.4 and 3.10 above.

Proposition 3.11. Let $g$ satisfy (3.1) and let $m$ be given by (2.17). An element $x$ belongs to $\operatorname{dom}(g(A))$ if and only if 
(i) $\lim _{t \rightarrow \infty} \operatorname{tm}(t) \mathrm{C}_{t}(A) x=0$

and

(ii) $\lim _{t \rightarrow \infty} \int_{0}^{t} s m^{\prime}(s) \mathrm{C}_{s}(A) x d s$ exists,

where the limits take place in either the strong or the weak topology of $X$.

Proof. Note that for all $x \in X$ and $t>0$,

$$
\int_{0}^{t} m(s) T(s) x d s=t m(t) \mathrm{C}_{t}(A) x-\int_{0}^{t} s m^{\prime}(s) \mathrm{C}_{s}(A) x d s .
$$

By Theorem 2.9, if

$$
\lim _{t \rightarrow \infty} \int_{0}^{t} m(s) T(s) x d s
$$

exists weakly then the same limit exists in the strong topology of $X$. Thus, by (3.17), it suffices to show that if the limit in (3.18) exists strongly then (i) and (ii) hold in the strong topology of $X$ since the opposite implication is straightforward. Using (2.17), we infer that

$$
g(1 / t) \geq \int_{0}^{t} e^{-s / t} m(s) d s \geq e^{-1} \operatorname{tm}(t), \quad t>0 .
$$

Since by assumption $\operatorname{ran}(A)$ is dense in $X$ and $x \in \operatorname{dom}(g(A))$, [18, Theorem 3.4 and Proposition 4.2] imply that $g(1 / t) \mathrm{C}_{t}(A) x \rightarrow 0, t \rightarrow \infty$, and then (3.19) yields (i). If (i) holds, then from (3.17) it follows that (ii) holds as well.

If $g(z)=z^{-\alpha}, \alpha \in(0,1)$, then we can derive a slightly stronger result which is a continuous counterpart of [12, Lemma 4.1].

Theorem 3.12. If $\alpha \in(0,1)$ then the following statements are equivalent:

(i) $x \in \operatorname{dom}\left(A^{-\alpha}\right)$;

(ii) $\lim _{t \rightarrow \infty} \int_{0}^{t} s^{\alpha-1} \mathrm{C}_{s}(A) x d s \quad$ exists;

(iii) weak- $\lim _{t \rightarrow \infty} \int_{0}^{t} s^{\alpha-1} \mathrm{C}_{s}(A) x d s$ exists.

Proof. Let us prove that (i) $\Leftrightarrow$ (ii). Since

$$
z^{-\alpha}=\frac{1}{\Gamma(\alpha)} \int_{0}^{\infty} s^{\alpha-1} e^{-s z} d s, \quad z>0
$$

$z^{-\alpha}$ is represented as in (2.17) with $m(s)=\frac{1}{\Gamma(\alpha)} s^{\alpha-1}, s>0$, and by Proposition 3.11 it suffices to prove that (ii) yields

$$
t^{\alpha} \mathrm{C}_{t}(A) x \rightarrow 0, \quad t \rightarrow \infty .
$$


To this aim note that if (ii) holds, then by Fubini's theorem,

$$
\begin{aligned}
\int_{t}^{2 t} s^{\alpha-1} \mathrm{C}_{s}(A) x d s= & \int_{t}^{2 t} s^{\alpha-2} d s \int_{0}^{t} T(\tau) x d \tau \\
& +T(t) \int_{0}^{t}(r+t)^{\alpha-2} \int_{0}^{r} T(\tau) x d \tau d r \\
= & \frac{\left(1-2^{\alpha-1}\right)}{1-\alpha} t^{\alpha} \mathrm{C}_{t}(A) x+T(t) \int_{0}^{t}(r+t)^{\alpha-2} r \mathrm{C}_{r}(A) x d r,
\end{aligned}
$$

where the last sum goes to zero as $t \rightarrow \infty$. Thus to prove (3.20) it suffices to show that

$$
\lim _{t \rightarrow \infty} \int_{0}^{t}(r+t)^{\alpha-2} r \mathrm{C}_{r}(A) x d r=0 .
$$

Setting

$$
G_{t}(r):=\frac{r^{2-\alpha}}{(r+t)^{2-\alpha}},(t>0 \text { is fixed }) \quad R(r):=\int_{r}^{\infty} s^{\alpha-1} \mathrm{C}_{s}(A) x d s, \quad r \geq 0
$$

where the second function is well-defined, continuously differentiable and bounded on $[0, \infty)$ by our assumption, write

$$
\int_{0}^{t}(r+t)^{\alpha-2} r \mathrm{C}_{r}(A) x d r=-G_{t}(t) R(t)+\int_{0}^{t} G_{t}^{\prime}(r) R(r) d r .
$$

We prove that both terms on the right-hand side of (3.21) converge to zero as $t \rightarrow$ $\infty$, and thus obtain the statement.

First note that for all $t>0$ and $r>0$,

$$
0<G_{t}(r) \leq 1, \quad r>0 ; \quad \lim _{t \rightarrow \infty} G_{t}(r)=0
$$

Hence, by our assumption, $\lim _{t \rightarrow \infty}\left\|G_{t}(t) R(t)\right\|=0$. To prove the convergence to zero of the other term note that

$$
G_{t}^{\prime}(r)=(2-\alpha)\left(\frac{r}{r+t}\right)^{1-\alpha} \frac{t}{(r+t)^{2}}
$$

is positive on $(0, \infty)$ for each $t>0$. Let $\epsilon>0$ be fixed. Then by assumption there exists $b=b(\epsilon)$ such that $\|R(t)\| \leq \epsilon$ if $t \geq b$. Now using (3.22) we have for large enough $t$

$$
\begin{aligned}
\left\|\int_{0}^{t} G_{t}^{\prime}(r) R(r) d r\right\| & \leq \int_{0}^{b} G_{t}^{\prime}(r)\|R(r)\| d r+\sup _{r \geq b}\|R(r)\| \int_{b}^{T} G_{t}^{\prime}(r) d r \\
& \leq \sup _{r \geq b}\|R(r)\| G_{T}(b)+\epsilon G_{t}(t)<2 \epsilon,
\end{aligned}
$$

and the statement follows.

The proof of (i) $\Leftrightarrow$ (iii) is analogous to the proof of (i) $\Leftrightarrow$ (ii), and is therefore omitted. 


\section{Optimality of domain conditions}

In this section we give a number of results showing that the inverse theorems on rates proved in Section 3 are optimal. The results will illustrate, in particular, that implications of the form

$$
\left\|C_{t}(A) x\right\|=\mathrm{O}(1 / g(1 / t)), \quad t \rightarrow \infty \quad \Rightarrow \quad x \in \operatorname{dom}(g(A)),
$$

are far from being true, in general, and the direct theorems on rates obtained in [18] cannot be inverted. Thus to get positive statements one has to add either "extra rate" or "extra range" assumptions as it was done above.

We start by introducing basic objects for constructing our examples. Let $L^{1}:=$ $L^{1}(1, \infty)$, and define a bounded operator $M$ on $L^{1}$ by

$$
(M u)(s):=\frac{u(s)}{s}, \quad u \in L^{1} .
$$

Note that $-M$ generates the contraction $C_{0}$-semigroup $(T(t))_{t \geq 0}$ given by

$$
(T(t) u)(s):=e^{-t / s} u(s), \quad t \geq 0 .
$$

Thus we have in particular

$$
\left(\mathrm{C}_{t}(M) u\right)(s)=\frac{1}{t} \int_{0}^{t} e^{-\tau / s} u(s) d \tau=\frac{s\left(1-e^{-t / s}\right)}{t} u(s),
$$

and

$$
t\left\|\mathrm{C}_{t}(M) u\right\|_{L^{1}}=\left\|w_{t} u\right\|_{L^{1}}, \quad w_{t}(s):=s\left(1-e^{-t / s}\right) \quad u \in L^{1} .
$$

A direct application of functional calculus rules reveals that for any Stieltjes function $g$ the operator $g(M)$ is of the form

$$
\begin{aligned}
& \operatorname{dom}(g(M))=\left\{u \in L^{1}: \int_{1}^{\infty} g(1 / s)|u(s)| d s<\infty\right\}, \\
& (g(M) u)(s)=g(1 / s) u(s), \quad \text { for a.e. } s \geq 1 .
\end{aligned}
$$

It will be convenient to introduce the following family of norms on $L^{1}$ :

$$
N_{t}(u):=\frac{1}{t} \int_{1}^{t} s|u(s)| d s+\int_{t}^{\infty}|u(s)| d s, \quad u \in L^{1}, \quad t \geq 1 .
$$

These norms are equivalent to the original norm on $L^{1}$ :

$$
t^{-1}\|u\|_{L^{1}} \leq N_{t}(u) \leq\|u\|_{L^{1}}, \quad u \in L^{1}, \quad t \geq 1 .
$$


Moreover, using the inequalities

$$
\begin{aligned}
\left(1-e^{-1}\right) \tau & \leq 1-e^{-\tau} \leq \tau, & & \tau \in(0,1), \\
1-e^{-1} & \leq 1-e^{-\tau} \leq 1, & & \tau \geq 1
\end{aligned}
$$

and (4.3), we obtain

$$
\left(1-e^{-1}\right) N_{t}(u) \leq\left\|C_{t}(M) u\right\|_{L^{1}} \leq N_{t}(u), \quad t>1, \quad u \in L^{1} .
$$

As in Section 3, we assume in this section that $g$ satisfy (3.1).

We first show that Theorem 3.4 is sharp in the sense that for $M$ defined by (4.1) and for a large class of $g$, properties (3.7) and $x \in \operatorname{dom}(g(M))$ are in fact equivalent.

Theorem 4.1. Let g satisfy (3.1). The condition

$$
\int_{1}^{\infty} \frac{\left|g^{\prime}(1 / t)\right|\left\|C_{t}(M) u\right\|_{L^{1}}}{t^{2}} d t<\infty
$$

holds for all $u \in \operatorname{dom}(g(M))$ if and only if $g$ is integrable on $(0,1)$ and there exists $c>0$ such that

$$
\frac{1}{t} \int_{0}^{t} g(\tau) d \tau \leq \operatorname{cg}(t), \quad t \in(0,1) .
$$

Proof. Assume that (4.7) holds for all $u \in \operatorname{dom}(g(M))$. Since the operator $g(M)$ is closed, $\left(\operatorname{dom}(g(M)),\|\cdot\|_{\operatorname{dom}(g(M))}\right)$ is a Banach space with the graph norm

$$
\|u\|_{\operatorname{dom}(g(M))}:=\|g(M) u\|_{L^{1}}+\|u\|_{L^{1}}=\int_{1}^{\infty}(g(1 / s)+1)|u(s)| d s .
$$

Consider a linear operator

$$
\begin{aligned}
G & : \operatorname{dom}(g(M)) \mapsto L^{1}\left((1, \infty), L^{1}\right) \\
G u & :=\frac{\left|g^{\prime}(1 / t)\right| C_{t}(M) u}{t^{2}}, \quad u \in \operatorname{dom}(g(M)) .
\end{aligned}
$$

From our assumption it follows that $G$ is well defined. By a standard argument (after passing to appropriate a.e. convergent subsequences) $G$ is closed. Therefore, by the closed graph theorem and (4.6), it follows that

$$
\int_{1}^{\infty} \frac{\left|g^{\prime}(1 / t)\right| N_{t}(u)}{t^{2}} d t \leq c\|u\|_{\operatorname{dom}(g(M))},
$$

for some constant $c>0$ not depending on $u \in \operatorname{dom}(g(M))$. 
Using (4.4) and Fubini's theorem we can write down the left-hand side of (4.9) as follows:

$$
\begin{aligned}
\int_{1}^{\infty} \frac{\left|g^{\prime}(1 / t)\right| N_{t}(u)}{t^{2}} d t & =\int_{1}^{\infty} \frac{\left|g^{\prime}(1 / t)\right|}{t^{2}}\left\{\frac{1}{t} \int_{1}^{t} s|u(s)| d s+\int_{t}^{\infty}|u(s)| d s\right\} d t \\
& =\int_{1}^{\infty}|u(s)| W_{g}(s) d s
\end{aligned}
$$

where

$$
W_{g}(s):=-s \int_{s}^{\infty} \frac{g^{\prime}(1 / t)}{t^{3}} d t-\int_{1}^{s} \frac{g^{\prime}(1 / t)}{t^{2}} d t .
$$

Integrating by parts and taking into account that $\lim _{\tau \rightarrow 0+} \tau g(\tau)=0$ (by the bounded convergence theorem) we infer that $g \in L^{1}(0,1)$ and for every $s \geq 1$

$$
W_{s}(g)=-s \int_{0}^{1 / s} g^{\prime}(\tau) \tau d \tau-\int_{1 / s}^{1} g^{\prime}(\tau) d \tau=s \int_{0}^{1 / s} g(\tau) d \tau-g(1) .
$$

Thus (4.9) is satisfied for all $u \in \operatorname{dom}(g(M))$ if and only if

$$
\int_{1}^{\infty} W_{g}(s)|u(s)| d s \leq c \int_{1}^{\infty}(g(1 / s)+1)|u(s)| d s, \quad u \in \operatorname{dom}(g(M)),
$$

where $W_{s}(g)$ is given by (4.10). In turn, (4.9) is equivalent to

$$
W_{g}(s) \leq c(g(1 / s)+1), \quad s \geq 1 .
$$

Indeed, it suffices to note that $\operatorname{dom}(g(M))$ contains all integrable functions with compact support. Writing down (4.11) for $u=1_{(a, b)}, 1 \leq a<b<\infty$, we obtain that

$$
\int_{a}^{b} W_{g}(s) d s \leq c \int_{a}^{b}(g(1 / s)+1) d s
$$

Hence

$$
F(s):=\int_{1}^{s}\left[c(g(1 / t)+1)-W_{g}(t)\right] d t, \quad s \geq 1,
$$

is an increasing function, and then $F^{\prime}(s)=c(g(1 / s)+1)-W_{g}(s) \geq 0, s \geq 1$.

Therefore, (4.12) can be rewritten as

$$
s \int_{0}^{1 / s} g(\tau) d \tau-g(1) \leq c(g(1 / s)+1), \quad s \geq 1,
$$

that is

$$
\frac{1}{t} \int_{0}^{t} g(\tau) d \tau \leq c g(t)+c+g(1), \quad t \in(0,1) .
$$

Since the function $g$ is decreasing on $(0, \infty)$, the last inequality is equivalent to (4.8) (in general with a new constant $c>0$ ). 
Remark 4.2. A natural question is what are the functions $g$ satisfying (4.8). To show that the class of such functions is quite large we note that if

$$
\tau^{\alpha} g(\tau) \text { is increasing on }(0,1)
$$

for some $\alpha \in(0,1)$, then (4.8) is satisfied, e.g., $g$ could be a power function. Indeed, if (4.13) holds, then

$$
\int_{0}^{t} g(\tau) d \tau \leq t^{\alpha} g(t) \int_{0}^{t} \frac{d \tau}{\tau^{\alpha}}=\frac{t}{1-\alpha} g(t), \quad t \in(0,1) .
$$

On the other hand, there are Stieltjes functions $g \sim(0,0, \mu)$ with $g(0+)=\infty$ for which (4.8) is not true. For instance, if $g(z):=\frac{z-1}{z \log z}$ (see Example 2.4b)) then $g \notin L^{1}(0,1)$.

Now we prove that Corollary 3.6 is optimal.

Theorem 4.3. Let g satisfy (3.1). The condition

$$
\int_{1}^{\infty} \frac{g(1 / t)\left\|C_{t}(M) u\right\|_{L^{1}}}{t} d t<\infty
$$

holds for all $u \in \operatorname{dom}(g(M))$ if and only if $g$ is integrable on $(0,1)$ and there exists $c>0$ such that

$$
\frac{1}{t} \int_{0}^{t} g(\tau) d \tau+\int_{t}^{1} \frac{g(\tau)}{\tau} d \tau \leq \operatorname{cg}(t), \quad t \in(0,1) .
$$

Proof. The proof is similar to that of Theorem 4.1 and will only be sketched. Note that

$$
\begin{aligned}
\int_{1}^{\infty} \frac{g(1 / t) N_{t}(u)}{t} d t & =\int_{1}^{\infty} \frac{g(1 / t) \mid}{t}\left\{\frac{1}{t} \int_{1}^{t} s|u(s)| d s+\int_{t}^{\infty}|u(s)| d s\right\} d t \\
& =\int_{1}^{\infty}|u(s)| V_{s}(g) d s, \\
\text { where } \quad V_{s}(g) & :=s \int_{0}^{1 / s} g(\tau) d \tau+\int_{1 / s}^{1} \frac{g(\tau)}{\tau} d \tau .
\end{aligned}
$$

Then by the argument analogous to that in the proof of Theorem 4.1, (4.15) is equivalent to the inequality

$$
V_{s}(g) \leq c(g(1 / s)+1), \quad s \geq 1
$$

for some constant $c>0$, which in turn is equivalent to (4.16). 
Remark 4.4. Observe that if there exist $\alpha, \beta \in(0,1)$ such that $0<\beta<\alpha<1$ and

$$
\tau^{\alpha} g(\tau) \text { is increasing on }(0,1), \tau^{\beta} g(\tau) \text { is decreasing on }(0,1) \text {, }
$$

then (4.16) holds. Indeed, in view of (4.14), it suffices to note that

$$
\int_{t}^{1} \frac{g(\tau)}{\tau} d \tau \leq t^{\beta} g(t) \int_{t}^{1} \frac{d \tau}{\tau^{1+\beta}} \leq \frac{g(t)}{\beta}, \quad t \in(0,1) .
$$

Example 4.5. Observe that the Stieltjes function $g(z)=(z-1)^{-1} \log z$ satisfies (4.8) but it does not satisfy (4.16). Indeed, if $t \in(0,1 / 2)$ then we have

$$
\frac{1}{t} \int_{0}^{t} \frac{\log \tau d \tau}{\tau-1} \leq-\frac{2}{t} \int_{0}^{t} \log \tau d \tau \leq 2 \frac{\log t}{t-1}=2 g(t) .
$$

On the other hand,

$$
\int_{t}^{1} \frac{\log \tau d \tau}{(\tau-1) \tau} \geq-\int_{t}^{1} \frac{\log \tau d \tau}{\tau}=\frac{\log ^{2} t}{2},
$$

and (4.16) is violated.

Thus if $g$ satisfies the conditions of Theorem 4.3 and $u \notin \operatorname{dom}(g(M))$ then the integral in (4.15) diverges. In this case (4.15) can hardly be written in the form of sup-norm estimates. To circumvent this drawback, we use the notion of slowly varying function. Recall that (see [32, Chapter 1]) a measurable function $\epsilon:(a, \infty) \mapsto(0, \infty), a \geq 0$, is called slowly varying (at infinity) if for all $\lambda>0$ one has $\lim _{t \rightarrow+\infty} \epsilon(\lambda t) / \epsilon(t)=1$. We proceed with a result which in a sense complements Theorem 4.3.

Theorem 4.6. Assume that $g$ satisfies (3.1) and that (4.13) holds. Let $\epsilon$ be a function slowly varying on $(g(1), \infty)$. Then the function

$$
y(s):=-\frac{g^{\prime}(1 / s)}{s^{2} g^{2}(1 / s) \epsilon(g(1 / s))}, \quad s>1
$$

is positive, belongs to $L_{1}$, and satisfies

$$
N_{t}(y)=\mathrm{O}\left(\frac{1}{g(1 / t) \epsilon(g(1 / t))}\right), \quad t \rightarrow \infty .
$$

Moreover, $y \in \operatorname{dom}(g(M))$ if and only if

$$
\int_{g(1)}^{\infty} \frac{d \tau}{\tau \epsilon(\tau)}<\infty
$$


Proof. Since $g(0+)=\infty$, by the change of variable $\tau=g(1 / s), \tau \in(g(1 / t), \infty)$, we obtain for any $t \geq 1$

$$
\int_{t}^{\infty} y(s) d s=-\int_{t}^{\infty} \frac{g^{\prime}(1 / s) d s}{s^{2} g^{2}(1 / s) \epsilon(g(1 / s))}=\int_{g(1 / t)}^{\infty} \frac{d \tau}{\tau^{2} \epsilon(\tau)} .
$$

Hence, using [32, item $1^{\circ}$, page 18], we infer that $y \in L_{1}$.

Similarly,

$$
\int_{1}^{\infty} g(1 / s) y(s) d s=-\int_{1}^{\infty} \frac{g^{\prime}(1 / s) d s}{s^{2} g(1 / s) \epsilon(g(1 / s))}=\int_{g(1)}^{\infty} \frac{d \tau}{\tau \epsilon(\tau)},
$$

and, in view of the description of $\operatorname{dom}(g(M))$ in (4.4), the statement follows.

It remains to prove that $y$ satisfies (4.19). Observe that by means of (4.4) and (4.21) one can rewrite (4.19) as

$$
\frac{1}{t} \int_{1}^{t} s y(s) d s \leq \frac{c}{g(1 / t) \epsilon(g(1 / t))}, \quad t \geq 1
$$

for some constant $c>0$. By [32, Section 1.5], for any $\delta>0$ the function $t^{-\delta} \epsilon(t)$ is equivalent as $t \rightarrow \infty$ to a positive function decreasing on $(g(1), \infty)$, and the function $t^{\delta} \epsilon(t)$ is equivalent as $t \rightarrow \infty$ to a positive function increasing on $(g(1), \infty)$. Therefore, since $g(0+)=\infty$ and $g$ is decreasing, for any $\delta>0, g^{-\delta}(\tau) \epsilon(g(\tau))$ is equivalent to a function increasing on $(1, \infty)$. Choose now positive $\delta$ such that $\beta:=(1+\delta) \alpha \in(0,1)$, where $\alpha$ is defined in (4.13). Then

$$
\tau^{\beta} g(\tau) \epsilon(g(\tau))=\left(\tau^{\alpha} g(\tau)\right)^{1+\delta} g^{-\delta}(\tau) \epsilon(g(\tau))
$$

is equivalent to a function increasing on $(0,1)$. In other words, the function $g(1 / s) \epsilon(g(1 / s))$ is equivalent to a measurable function $\psi$ such that $s^{\beta} / \psi(s)$ is increasing on $(1, \infty)$. Hence since $\tau\left|g^{\prime}(\tau)\right| \leq g(\tau), \tau>0$, by (3.12), we obtain for every $t \geq 1$

$$
\begin{aligned}
\frac{1}{t} \int_{1}^{t} s y(s) d s & =\frac{1}{t} \int_{1}^{t} \frac{\left|g^{\prime}(1 / s)\right| d s}{s g^{2}(1 / s) \epsilon(g(1 / s))} \leq \frac{1}{t} \int_{1}^{t} \frac{d s}{g(1 / s) \epsilon(g(1 / s))} \\
& \leq \frac{c}{t} \int_{1}^{t} \frac{s^{\beta} d s}{s^{\beta} \psi(s)} \leq \frac{c}{t} \frac{t^{\beta}}{\psi(t)} \int_{1}^{t} \frac{d s}{s^{\beta}} \\
& \leq C \frac{t^{\beta}}{\operatorname{tg}(1 / t) \epsilon(g(1 / t))} \int_{1}^{t} \frac{d s}{s^{\beta}} \leq C \frac{1}{(1-\beta) g(1 / t) \epsilon(g(1 / t))}
\end{aligned}
$$

where $c, C$ are positive constants, and the proof is complete.

Theorem 4.6 and (4.6) imply the following statement ( $c f$. Corollary 3.5). 
Corollary 4.7. Assume that functions $g$ and $\epsilon$ satisfy the conditions of Theorem 4.6. If

$$
\int_{g(1)}^{\infty} \frac{d \tau}{\tau \epsilon(\tau)}=\infty
$$

then there exists $y \in L^{1}$ such that

$$
\left\|\mathrm{C}_{t}(M) y\right\|_{L^{1}}=\mathrm{O}\left(\frac{1}{g(1 / t) \epsilon(g(1 / t))}\right), \quad t \rightarrow \infty,
$$

but $y \notin \operatorname{dom}(g(M))$.

Remark 4.8. By $[18$, Theorem 4.6] if $\epsilon:(0, \infty) \rightarrow(0, \infty)$ is an increasing function such that $\lim _{t \rightarrow \infty} \epsilon(t)=\infty$, then there exists $x \in \operatorname{dom}(g(M))$ such that

$$
\sup _{t \geq 1} g(1 / t) \epsilon(g(1 / t))\left\|\mathrm{C}_{t}(M) x\right\|_{L^{1}}=\infty .
$$

Thus conditions like (4.23) cannot hold for all elements of the corresponding domain.

Example 4.9. Observe that $g(z)=z^{-\gamma}, \gamma \in(0,1)$ is a Stieltjes function satisfying (4.13) for $\alpha \in(\gamma, 1)$. Therefore, $g$ satisfies also (4.8). (The latter fact can also be checked directly.) Since

$$
\epsilon(s):=\log (s+2) \log (\log (s+3)), \quad s \geq 0
$$

is slowly varying on $(0, \infty)$, the functions $g$ and $\epsilon$ satisfy the conditions of Theorem 4.1. Hence, by Corollary 4.7 there exists

$$
y \in L^{1}, \quad y \notin \operatorname{dom}\left(M^{-\gamma}\right)
$$

such that

$$
\left\|\mathrm{C}_{t}(M) y\right\|_{L^{1}}=\mathrm{O}\left(\frac{1}{t \gamma \log (t) \log (\log t)}\right), \quad t \rightarrow \infty .
$$

Example 4.10. Note that the Stieltjes function $g(z)=\log \left(1+z^{-1}\right)$ and the function $\epsilon$ defined by (4.24) satisfy the conditions of Theorem 4.6 (since $g$ satisfies (4.13) for any $\alpha \in(0,1))$. Hence, by Corollary 4.7 , there exists

$$
y \in L^{1}, \quad y \notin \operatorname{dom}\left(\log \left(I+M^{-1}\right)\right),
$$

such that

$$
\left\|\mathrm{C}_{t}(M) y\right\|_{L^{1}}=\mathrm{O}\left(\frac{1}{\log t[\log (\log t) \log (\log (\log t))]}\right), \quad t \rightarrow \infty .
$$




\section{Appendix}

Recall that if $(T(t))_{t \geq 0}$ is a bounded $C_{0}$-semigroup on $X$ then for each $x \in X \backslash\{0\}$ the Cesáro means $\mathrm{C}_{t}(A) x$ cannot decay faster than $1 / t$ as $t \rightarrow \infty$. The proposition below shows that it is not possible to 'improve' this extremal rate of decay of $\mathrm{C}_{t}(A) x$ by requiring the smallness of $\mathrm{C}_{t}(A) x$ in an integral sense.

Proposition 5.1. Let $(T(t))_{t \geq 0}$ be a bounded $C_{0}$-semigroup on a Banach space $X$ with generator $-A$. If for $x \in X$ there exists $\left\{t_{k}: k \geq 1\right\} \subset(0, \infty), t_{k} \rightarrow \infty$, $k \rightarrow \infty$, such that

$$
\text { weak }-\lim _{k \rightarrow \infty} \frac{1}{t_{k}} \int_{0}^{t_{k}} s \mathrm{C}_{s}(A) x d s=0
$$

then $x=0$. In particular, if

$$
\text { weak }-\lim _{t \rightarrow \infty} t \mathrm{C}_{t}(A) x=0
$$

then $x=0$.

Proof. Since

$$
t A(I+A)^{-1} \mathrm{C}_{t}(A) x=(I-T(t))(I+A)^{-1} x, \quad t>0,
$$

we have

$$
\left[A(I+A)^{-1}\right]^{2} \frac{1}{t} \int_{0}^{t} s \mathrm{C}_{s}(A) x d s=A(I+A)^{-2} x-\frac{(I-T(t))}{t}(I+A)^{-2} x .
$$

As the operator $A(I+A)^{-1}$ is bounded, the latter equality and (5.1) imply that $A(I+A)^{-2} x=0$ and then $x \in \operatorname{ker} A$. But if $x \in \operatorname{ker} A$ then

$$
\frac{1}{t} \int_{0}^{t} s \mathrm{C}_{s}(A) x d s=\frac{1}{t} \int_{0}^{t} s d s x=\frac{t}{2} x
$$

and, using (5.1) once again, we conclude that $x=0$.

Theorem 5.2. Let $(T(t))_{t \geq 0}$ be a bounded $C_{0}$-semigroup on a Banach space $X$ with generator $-A$. Let $\varphi$ be a positive and increasing on $[1, \infty)$ function such that

$$
\int_{1}^{\infty} \frac{d t}{t \varphi(t)}=\infty
$$

If $x \in X$ satisfies

$$
\int_{1}^{\infty} \frac{\left\|\mathrm{C}_{t}(A) x\right\|}{\varphi(t)} d t<\infty
$$

then $x=0$. In particular, if

$$
\int_{1}^{\infty} \frac{\left\|\mathrm{C}_{t}(A) x\right\|}{\log (1+t)} d t<\infty
$$

then $x=0$. 
Proof. Define

$$
\Theta(t):=\int_{0}^{t} s\left\|C_{s}(A) x\right\| d s, \quad t \geq 1 .
$$

If $s>1$, then

$$
\begin{aligned}
\int_{1}^{s} \frac{\left\|C_{t}(A) x\right\|}{\varphi(t)} d t & =\int_{1}^{s} \frac{1}{t \varphi(t)} d \Theta(t) \\
& =\frac{\Theta(s)}{s \varphi(s)}-\frac{\Theta(1)}{\varphi(1)}-\int_{1}^{s} \Theta(t) d\left(\frac{1}{t \varphi(t)}\right) \\
& =\frac{\Theta(s)}{s \varphi(s)}-\frac{\Theta(1)}{\varphi(1)}+\int_{1}^{s} \frac{\Theta(t)}{t^{2} \varphi(t)} d t-\int_{1}^{s} \frac{\Theta(t)}{t} d\left(\frac{1}{\varphi(t)}\right) \\
& \geq-\frac{\Theta(1)}{\varphi(1)}+\int_{1}^{s} \frac{\Theta(t)}{t^{2} \varphi(t)} d t .
\end{aligned}
$$

Hence by (5.3) it follows that

$$
\int_{1}^{\infty} \frac{\Theta(t) d t}{t^{2} \varphi(t)}<\infty
$$

Therefore by (5.2) and (5.4) we infer that there exists $t_{k} \rightarrow \infty, k \rightarrow \infty$, such that $\lim _{k \rightarrow \infty} \Theta\left(t_{k}\right) / t_{k}=0$. Therefore (5.1) holds and by Proposition 5.1 we have $x=0$.

Remark 5.3. Note that if $g$ as in Section 3 and $g \sim(0, b, \mu), b>0$, then $g^{\prime}(1 / t) t^{-2}$ and $g(1 / t) / t$ are separated from zero on $(0, \infty)$ so that the conditions $(3.7)$ and (3.11) reduce to (5.3) with $\varphi(t) \equiv 1$ yielding $x=0$.

\section{References}

[1] I. ASSANI and M. LIN, On the one-sided ergodic Hilbert transform, In: "Ergodic Theory and Related Fields", I. Assani (ed.) Contemporary Math., Vol. 430, Providence, RI, 2007, 21-39.

[2] F. E. BROWDER, On the iteration of transformations in noncompact minimal dynamical systems, Proc. Amer. Math. Soc. 9 (1958), 773-780.

[3] P. BUtZER and A. GESSINGER, Ergodic theorems for semigroups and cosine operator functions at zero and infinity with rates; applications to partial differential equations. A survey, In: "Mathematical Analysis, Wavelets, and Signal Processing", Contemporary Math. 190 (1995), 67-94.

[4] P. BUtZer and U. WestPhAL, The mean ergodic theorem and saturation, Indiana Univ. Math. J. 20 (1971), 1163-1174.

[5] G. CohEn, C. CUNY and M. LIN, The one-sided ergodic Hilbert transform in Banach spaces, Studia Math. 196 (2010), 251-263.

[6] G. Cohen, C. CUNY and M. Lin, On convergence of power series of $L_{p}$ contractions, Banach Center Publications, to appear.

[7] G. COHEN and M. LIN, Laws of large numbers with rates and the one-sided ergodic Hilbert transform, Illinois J. Math. 47 (2003), 997-1031. 
[8] G. CoHEn and M. LIN, Extensions of the Menchoff-Rademacher theorem with applications to ergodic theory, Israel J. Math. 148 (2005), 41-86.

[9] G. COHEN and M. LIN, The one-sided ergodic Hilbert transform of normal contractions, In: "Characteristic Functions, Scattering Functions and Transfer Functions", the Moshe Livšic memorial volume, Birkhäuser, Basel, 2009, 77-98.

[10] C. Cuny, Pointwise ergodic theorems with rate and with applications to limit theorems for stationary processes, Stoch. Dyn. 11 (2011), 135-155.

[11] C. CUNY, On the a.s. convergence of the one-sided ergodic Hilbert transform, Ergodic Theory Dynam. Systems 29 (2009), 1781-1788.

[12] C. CUNY, Norm convergence of some power series of operators in $L^{p}$ with applications in ergodic theory, Studia Math. 200 (2010), 1-29.

[13] C. CUNY and M. LIN, Pointwise ergodic theorems with rate and application to the CLT for Markov chains, Ann. Inst. Henri Poincaré, Probab. Stat. 45 (2009), 710-733.

[14] R. DeLaubenfels, Automatic extensions of functional calculi, Studia Math. 114 (1995), 237-259.

[15] Y. DERRIENNIC, Some aspects of recent works on limit theorems in ergodic theory with special emphasis on the "central limit theorem", Discrete Contin. Dyn. Syst. 15 (2006), $143-158$.

[16] Y. DERRIENNIC and M. LIN, Fractional Poisson equations and ergodic theorems for fractional coboundaries, Israel J. Math. 123 (2001), 93-130.

[17] A. Gomilko, M. HAASE and Yu. Tomilov, On rates in mean ergodic theorems, Math. Res. Letters 18 (2011), 201-213.

[18] A. Gomilko, M. HaAse and Yu. Tomilov, Bernstein functions and rates in mean ergodic theorems for operator semigroups, J. Anal. Math. 118 (2012), 545-576.

[19] M. HAAse, "The Functional Calculus for Sectorial Operators", Operator Theory: Advances and Applications, Vol. 169, Birkhäuser, Basel, 2006.

[20] M. HAASE and YU. TOMILOV, Domain characterizations of certain functions of powerbounded operators, Studia Math. 196 (2010), 265-288.

[21] E. Hille and R. S. Phillips, "Functional Analysis and Semi-Groups", $3^{\text {rd }}$ printing of rev. ed. of 1957, Colloq. Publ., Vol. 31, Amer. Math. Soc., Providence, RI, 1974.

[22] F. HIRSCH, Intégrales de résolvantes et calcul symbolique, Ann. Inst. Fourier (Grenoble) 22 (1972), 239-264.

[23] F. HIRSCH, Domaines d'opérateurs représentés comme intégrales de résolvantes, J. Funct. Anal. 23 (1976), 199-217.

[24] N. JACOB, "Pseudo Differential Operators and Markov Processes, Vol. I. Fourier Analysis and Semigroups", Imperial College Press, London, 2001.

[25] A. G. KACHUROVSKII, Rates of convergence in ergodic theorems, Uspekhi Mat. Nauk 51 (1996), 73-124 (Russian); Russian Math. Surv. 51 (1996), 653-703.

[26] A. G. KACHUROVSKII and A. V. RESHETENKO, On the rate of convergence in von Neumann's ergodic theorem with continuous time, Mat. Sb. 201 (2010), 25-32 (Russian); Sb. Math. 201 (2010), 493-500.

[27] U. Krengel, "Ergodic Theorems", de Gruyter Studies in Math. 6, Walter de Gruyter, Berlin, 1985.

[28] R. S. PHILLIPS, On the generation of semigroups of linear operators, Pacific J. Math. 2 (1952), 343-369.

[29] S.-Y. SHAW, Convergence rates of ergodic limits and associated solutions, J. Approx. Theory 75 (1993), 157-166.

[30] R. L. SCHILling, Subordination in the sense of Bochner and a related functional calculus, J. Austral. Math. Soc. 64 (1998), 368-396.

[31] R. SCHILling, R. SONG and Z. VONDRAČEK, "Bernstein Functions", de Gruyter Studies in Math. 37, Walter de Gruyter, Berlin, 2010.

[32] E. Seneta, "Regularly Varying Functions", Lecture Notes in Math., Vol. 508, Springer, 1976. 
[33] U. WeSTPHAL, A generalized version of the Abelian mean ergodic theorem with rates for semigroup operators and fractional powers of infinitesimal generators, Results Math. 34 (1998), 381-394.

Faculty of Mathematics and Computer Science Nicholas Copernicus University

ul. Chopina 12/18

87-100 Toruń, Poland

and

Institute of Telecommunications

and Global Information Space

National Academy of Sciences of Ukraine

Kiev, Ukraine

gomilko@mat.umk.pl

Faculty of Mathematics and Computer Science Nicholas Copernicus University

ul. Chopina 12/18

87-100 Toruń, Poland

and

Institute of Mathematics

Polish Academy of Sciences

Śniadeckich 8

00-956 Warsaw, Poland

tomilov@mat.umk.pl 